\title{
Inter-disciplinary approach to the management of central giant cell lesion- A case report
}

\author{
Harneet Kaur $^{1, *}$, Jitender Dabas ${ }^{2}$, Rudra Deo Kumar ${ }^{3}$, Sujata Mohanty ${ }^{4}$ \\ ${ }^{1}$ Assistant Professor, Dept. of Orthodontics and Dentofacial Orthopaedics, Jamia Millia Islamia, New Delhi, ${ }^{2}$ Senior Resident, \\ ${ }^{3}$ Post Graduate Resident, ${ }^{4}$ Professor and Head, Dept. of Oral \& Maxillofacial Surgery, Maulana Azad Institute of Dental \\ Sciences, New Delhi, India
}

*Corresponding Author:

Email: dr.harneetkaur@gmail.com

\begin{abstract}
Central giant cell Lesion (CGCL) of the jaw is a relatively uncommon benign lesion of unknown etiology. Essentially a destructive process, the most common treatment is surgical removal; however, alternative therapies (intra-lesional injections of corticosteroids, interferon alpha, and calcitonin) have been used in order to avoid undesirable damage to the jaws and teeth. Clinically along with facial asymmetry, the lesion often results in deranged occlusion that must be treated orthodontically. This case report illustrates sequential management of CGCL with intra-lesional corticosteroids followed by surgical re-contouring along with orthodontic considerations in management of associated malocclusion. Difficulties in handling such cases and projecting the recurrence of CGCL shall also be discussed.
\end{abstract}

Keywords: Central giant cell lesion, Buccal cross-bite, Intra-lesional corticosteroids, Orthodontic treatment.

\section{Introduction}

The central giant cell lesion (CGCL) of the jaws is a relatively uncommon pathology with variable and impulsive biologic behaviour and accounting for less than $7 \%$ of all benign lesions. First described by Jaffe in 1953 as a giant-cell reparative granuloma of the jaw bones, ${ }^{1}$ the lesion occurs twice more frequently in females than in males with greater propensity in mandible $(80 \%)$ than in the maxilla. ${ }^{2-5}$ The age of most patients ranges from 10 to 25 years with a peak incidence between 10-14 years for males and between 15 and 19 years for females. ${ }^{5}$

The lesion represents essentially represents a destructive process, ${ }^{3,6}$ The etiology of which is obscure except that trauma has sometimes been mentioned as an important factor. Clinically and radiographically the lesion should be distinguished from ameloblastoma, traumatic bone cyst, adenomatoid odontogenic tumour and fibrous dysplasia. ${ }^{7}$ With most common chief complaint of pain and swelling of the jaws resulting in facial asymmetry, there is difficulty in executing normal mastication on the affected site. Intra-orally, although overlying mucosa is occasionally ethythematous, the lesions results in deranged occlusion with displacement of teeth, root resorption and mobility of teeth if with severe destruction of alveolar bone bone. ${ }^{2,8,9}$ Radiologically, CGCL appears as a clearly delineated bony lesion with loss of lamina dura around teeth. The lesion may be unilocular or multilocular, presenting with varying degrees of expansion of the cortical plates. ${ }^{6-8}$

This case report describes sequential management of CGCL with intra-lesional corticosteroids followed by surgical re-contouring and post-surgical orthodontics for management of associated malocclusion.

Diagnosis and Etiology: A 14-year old female reported with progressive swelling of right side of lower jaw since past 3 months. No positive history of trauma could be elicited from the patient. Upon examination, there was a clear asymmetry of posterior body and angle region on the right side of mandible. The lesion was approximately $5 \times 4 \mathrm{~cm}$ in size and tender and soft on palpation. There was no paresthesia over the lower lip. Intra-orally, there was lingual tilting of molars on the affected side. OPG \& CT scan revealed multilocular radiolucency with wispy opacification in relation to right body and angle of mandible. Aspiration of the lesion yielded frank blood. Incisional biopsy performed and diagnosis was confirmed to CGCL right mandible. (Fig. 1)

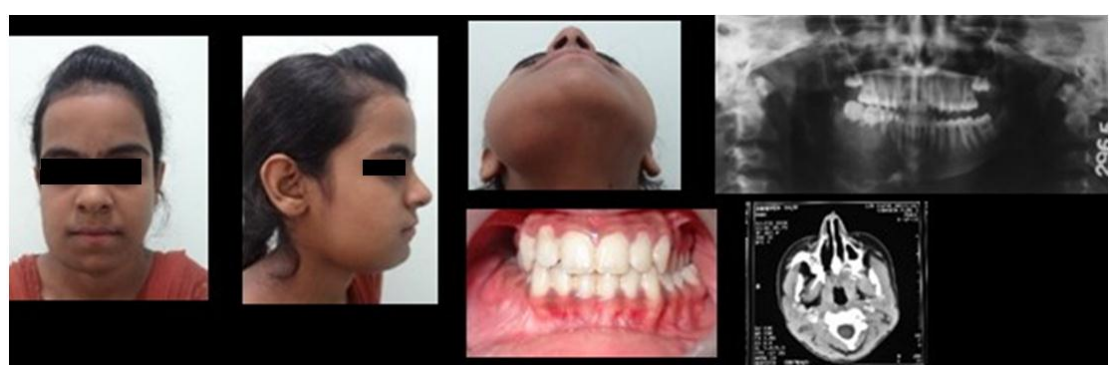

Fig. 1: Pre-treatment Photographs, OPG and CT scan 
Treatment Objectives: The treatment objectives were to intercept the progression of the swelling and correct facial asymmetry. Thereafter, to orthodontically correct posterior lingual cross-bite on right buccal segments and to provide a proper functional occlusion.

Treatment Alternatives: Considering age of the patient and aspiration of blood from the lesion, it was decided to start the treatment with less invasive approach of intra-lesional corticosteroids/ calcitonin followed by surgical contouring the bone (if required) and orthodontic correction of displaced teeth to achieve proper functional occlusion.

Treatment Progress: Treatment was commenced with injections of intralesional triamcinolone $(10 \mathrm{mg} / \mathrm{ml})$ mixed with $2 \%$ lignocaine and 1:200,000 adrenaline in 1:1 ratio. $1 \mathrm{ml}$ of this solution was injected for every 1 $\mathrm{cm}^{3}$ as calculated on OPG. Weekly injections were given for 10 weeks, by the end of which the lesion site became sclerosed and it was no longer possible to penetrate inside the lesion.

At eighteen months follow-up after the last injection, the lesion was consolidated with a single nodular diffuse swelling over the right angle of mandible that was non-tender and bony hard on palpation. (Fig. 2). OPG x-ray revealed mixed osteolytic sclerotic lesion of right posterior body and angle of mandible with root resorption of first and second molars. Surgical resection of expanded bone followed by recontouring and smoothening was performed. The patient was followed up clinically and radiologically at regular intervals, and the bony defect was almost completely resolved within about 14 months of the surgery.

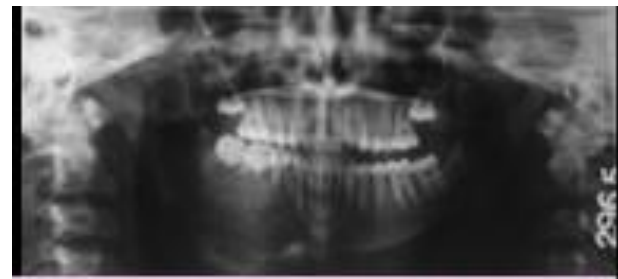

PRE-TREATMENT OPG

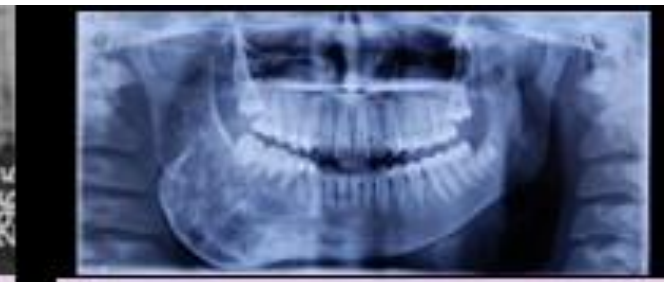

PRE-SURGICAL OPG

Fig. 2: Comparison of pre-treatment and pre-surgical OPG

Two years post-operatively, there was no evidence of any recurrence and the patient was considered for orthodontic correction of displaced teeth. (Fig. 3)

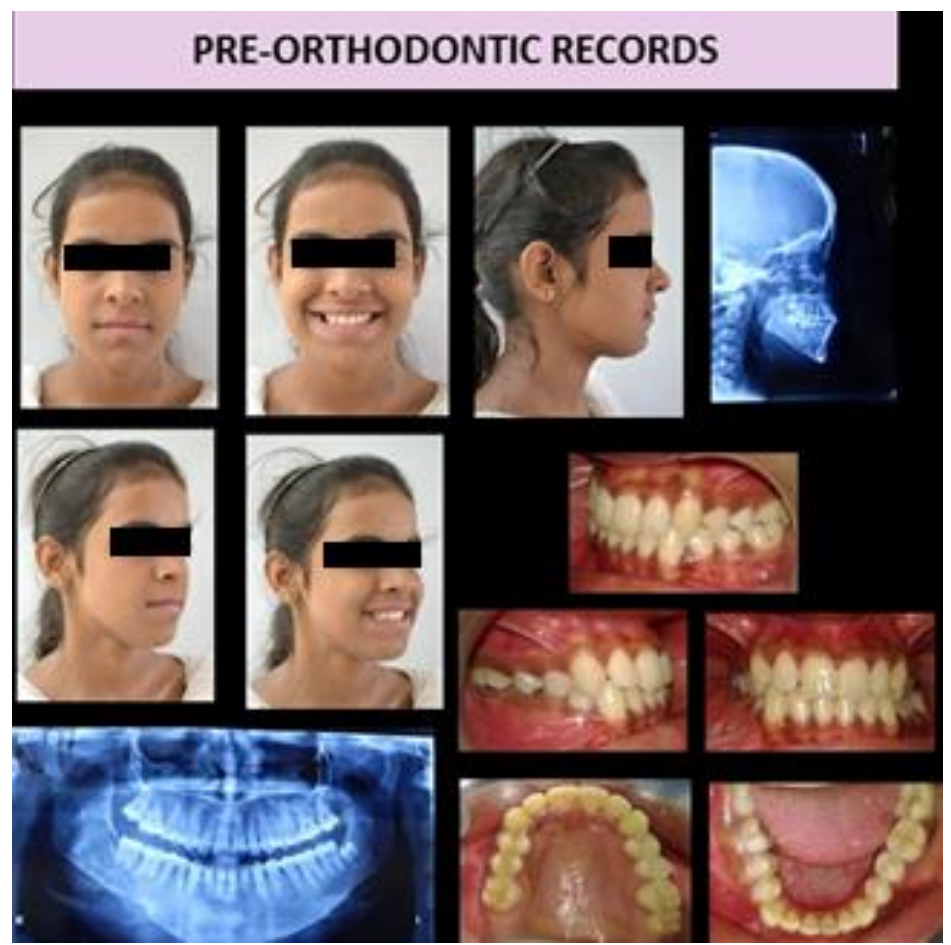

Fig. 3: Pre-Orthodontic treatment records 
Upon examination, patient had a relatively symmetric mesoprosopic face with straight profile, normal growth pattern, average nasolabial angle and competent lips. Smile evaluation revealed increased right buccal corridor space. Intra-oral examination revealed Angle's Class I type 4 malocclusion with lingual canting mandibular right second premolars and molars leading to buccal cross bite (scissor's bite).

The orthodontic treatment plan was to upright the lingually displaced teeth using cross-elastics along with
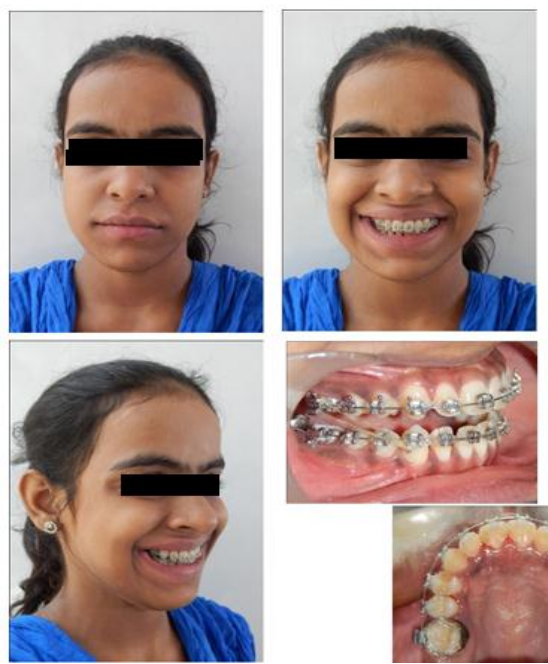

conventional straightwire mechanics. After the maxillary arch was aligned and stabilized with .019X.025" stainless steel wire, posterior bite-block were given on left mandibular molars and on right side, inter-maxillary cross elastics were started to upright the mandibular molars (Fig. 4). Additional trans-palatal arch was given for cross-arch stabilization. Once the desired uprighting of teeth was achieved, remaining mandibular arch was bonded for alignment, torquing and occlusal settling.

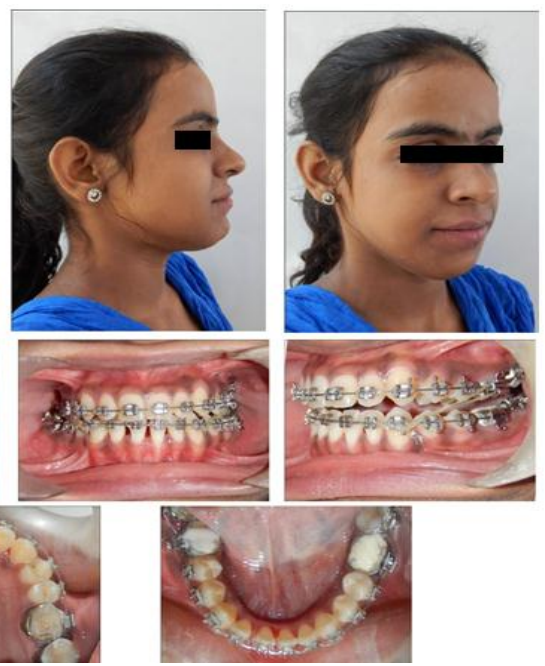

Fig. 4: Treatment progress with inter-maxillary cross elastics

\section{Results}

After 25 months of active treatment, all fixed appliances were removed, maxillary and mandibular removable retainers were placed to maintain the dental correction achieved. (Fig. 5) Periodic follow-up at every 6-months was planned to monitor the stability of occlusion and any progression of lesion (Fig. 6)

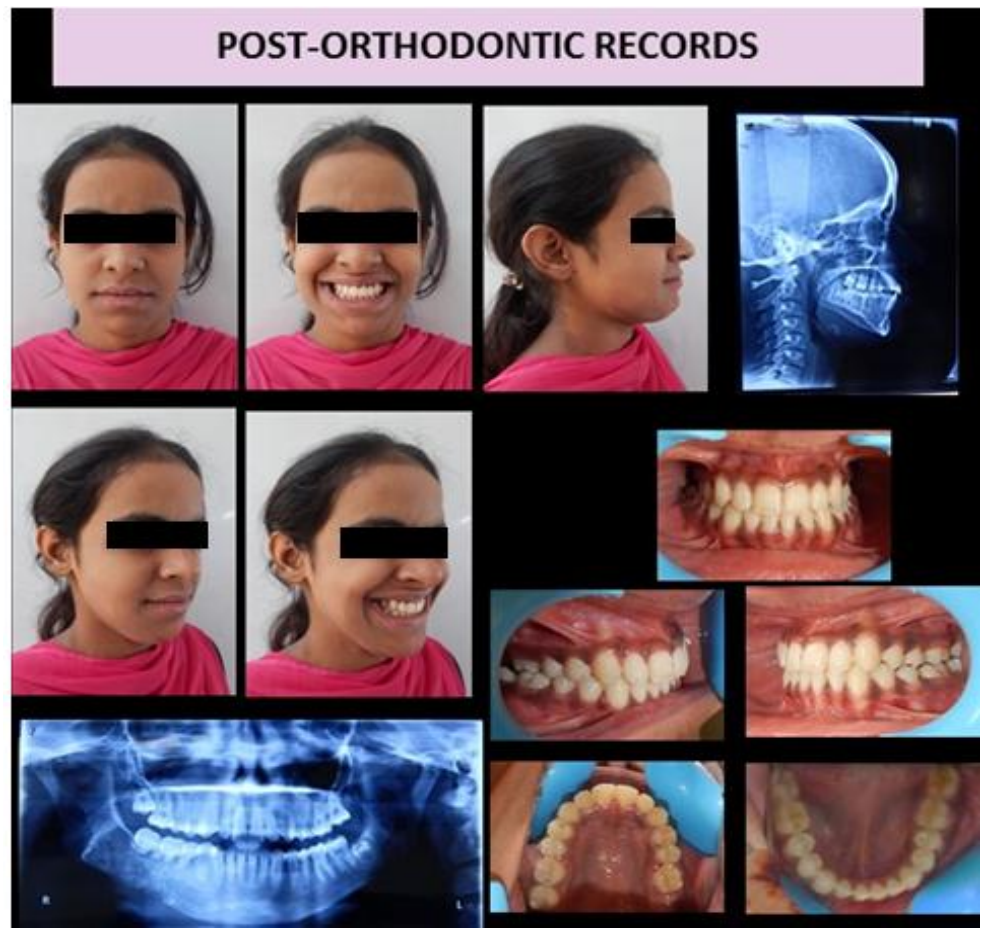

Fig. 5: Post-Orthodontic treatment records 


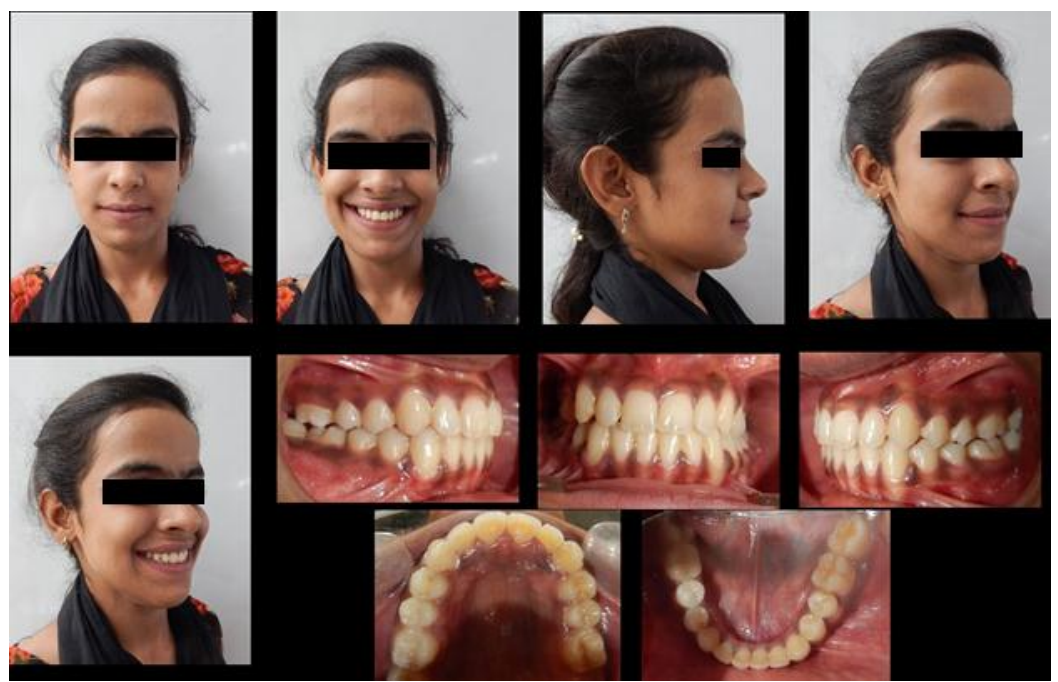

Fig. 6: 1-year post-retention photographs

\section{Discussion}

CGCL usually presents as a painless, slow growing swelling of the jaw. Presence of a fine opacification within the lesion is the most significant radiographic sign associated with CGCL. Aspiration of blood and histopathologic presence of multinucleated giant cells further confirmed the diagnosis.

The treatment was commenced with less invasive approach for intra-lesional corticosteroids. The patient also didn't present with any systemic contraindication to steroid administration like local infection, diabetes, peptic ulcer or known allergy to steroids. Corticosteroids have relatively higher success rate as compared to calcitonin /interferon- $\alpha .{ }^{10}$ The lesion was responsive to this therapy as increasing backpressure and difficulty in penetration of needle into the lesion was noted upon weekly injections. The lesion became hard and painless. At 18 months follow-up, there was no noticeable change in size of the swelling. OPG showed increased radio-opacity within the lesion. Considering the benign, non-progressive nature of the lesion, surgical re-contouring was done to correct facial asymmetry.

CGCL is frequently associated with malocclusion due to displacement of teeth and tooth germ, root resorption and cortical perforation. ${ }^{5} \mathrm{~A}$ more aggressive CGCL may cause resorption of adjacent roots, pain, or perforation of the overlying bone, and tends to recur after curettage. ${ }^{11}$ Hence it usually necessitates delaying orthodontic treatment until the bone heals. Also, histologically the lesion requires many months to resolve and complete remodelling is very slow. In this case, we delayed orthodontic treatment with fixed appliances until bony repair had taken place considering the possible interference of bone healing by tooth movement.

The most important aspect orthodontists should keep in mind is recurrence of the lesion. ${ }^{8,11}$ It is advisable to perform sequential tooth movement with light forces. In this case, buccal cross-bite developed due to lingual displacement of mandibular molars. We considered progressive uprighting of the molars with cross-elastics first, followed by torqueing with rectangular archwires. Also regular radiological screening at various stages of treatment was done due to rule out the possibility of recurrence.

Another feature for consideration is root resorption. Although blunting of root apices was there in this case, but the teeth also responded well to orthodontic forces and no further progression of root resorption was noted. Also the good alveolar support with a proper gingival attachment height was accomplished after orthodontic alignment.

\section{Conclusion and Recommendations:}

Although CGCLs are relatively uncommon, these lesions should be considered by surgeons as well as orthodontists as part of a differential diagnosis of patients with grossly displaced teeth.

Following points can be considered as orthodontist's checklist for management of CGCL

1. Orthodontic treatment should be considered only after complete ossification of wound site.

2. Most important aspect orthodontists should keep in mind is the chances of recurrence of the lesion.

3. Care should be taken to keep orthodontic forces as gentle as possible.

4. Perform regular screening to rule out the possibility of recurrence.

5. Root resorptions of the involved teeth must be monitored and endodontic intervention can be performed if required.

6. Successful alignment along with good alveolar bone regeneration is mandatory for long-term treatment stability.

Due to difficulty in projecting the recurrence of CGCG and long-term stability of occlusion, it is advisable to review such a patient periodically for any clinical and/or radiographic changes. 


\section{References}

1. Jaffe HL. Giant-cell reparative granuloma, traumatic bone cyst, and fibrous (fibro-osseous) dysplasia of the jawbones. Oral Surg 1953;6:159-75.

2. Sidhu MS, Parkash H, Sidhu SS. Central giant cell granuloma of the jaws - review of 19 cases. Br J Oral Maxillofac Surg 1995;33:43-6.

3. Cohen MA, Hertzanu Y. Radiologic features, including those seen with computed tomography of central giant cell granuloma of the jaws. Oral Surg Oral Med Oral Pathol 1988;65:255-261.

4. Waldron C, Shafer WG. The central giant cell reparative granuloma of the jaws. Am J Clin Pathol 1966;45:437-44.

5. De Lange J, Van den Akker HP, Van den Berg H. Central giant cell granuloma of the jaw: a review of the literature with emphasis on therapy options. Oral Surg Oral Med Oral Pathol Oral Radiol Endod 2007;104:603-15.

6. Shafer WG, Hine MK, Levy BM. A Textbook of Oral Pathology 4th ed. WB Saunders: Philadelphia, 1983;146149.

7. Kerly TR, Schow CE. Central giant cell granuloma or cherubism. Oral Surg Oral Med Oral Pathol 1981;51:128-130.

8. Romero M, Romance A, Garcia-Recuero JI, Fernández A. Orthopedic and Orthodontic Treatment in Central Giant Cell Granuloma Treated With Calcitonin. Cleft Palate Craniofac J. 2011;48(5):519-525.

9. Leonard TJ, McNamara C, McNamara CM. CASE REPORT Management of an Unerupted Canine Associated with a Central Giant Cell Granuloma. J Clin Orthod 2003;37(2):75-8.

10. Mohanty S, Jhamb A. Central giant cell lesion of mandible managed by intralesional triamcinolone injections. A report of two cases and literature review. Med Oral Patol Oral Cir Bucal 2009;14(2):e98-102.

11. Allen, D.T. and Sheats, R.D. A central giant cell granuloma in a patient seeking orthodontic treatment. $J$ Am Dent Assoc 2001;132:1255-1260,. 